\title{
Pharmacological Inhibition of Cryptochrome and REV-ERB Promotes DNA Repair and Cell Cycle Arrest in Cisplatin-Treated Human Cells
}

Nadeen Anabtawi

Wright State University

William Cvammen

Wright State University

Michael G. Kemp ( $\nabla$ mike.kemp@wright.edu )

Wright State University

\section{Research Article}

Keywords: Circadian clock, DNA repair, cell cycle, cisplatin, cell proliferation, pharmacology

Posted Date: July 30th, 2021

DOI: https://doi.org/10.21203/rs.3.rs-751809/v1

License: (c) (i) This work is licensed under a Creative Commons Attribution 4.0 International License.

Read Full License 


\section{Abstract}

Nucleotide excision repair (NER) and cell cycle checkpoints impact the ability of the anti-cancer drug cisplatin to inhibit cell proliferation and induce cell death. Genetic studies have shown that both NER and cell cycle progression are impacted by the circadian clock, which has emerged as a novel pharmacological target for the treatment of various disease states. In this study, cultured human cell lines were treated with combinations of cisplatin and the circadian clock modulating compounds KS15 and SR8278, which enhance circadian clock transcriptional output by inhibiting the activities of the cryptochrome and REV-ERB proteins, respectively. Treatment of cells with KS15 and SR8278 protected cells against the anti-proliferative effects of cisplatin and increased the expression of NER factor XPA and cell cycle regulators Wee1 and p21 at the mRNA and protein level. Correlated with these molecular changes, KS15 and SR8278 treatment resulted in fewer unrepaired cisplatin-DNA adducts in genomic DNA and a higher fraction of cells in the $\mathrm{G} 1$ phase of the cell cycle. Thus, the use of pharmacological agents targeting the circadian clock could be a novel approach to modulate the responses of normal and cancer cells to cisplatin chemotherapy regimens.

\section{Introduction}

Cisplatin and other platinating agents are widely used in the treatment of different cancers due to their formation of adducts on DNA that interfere with DNA replication and induce cell death in rapidly proliferating cancer cells $s^{1,2}$. The nucleotide excision repair (NER) system plays an essential role in removing intra-strand cisplatin-DNA adducts from the genome ${ }^{3,4}$ and therefore limits the effectiveness of cisplatin chemotherapy regimens. Cell cycle checkpoints and other components of the cellular DNA damage response also impact cisplatin responses by allowing additional time for DNA repair prior to the entry of cells into $S$ phase and mitosis ${ }^{5,6}$, during which time unrepaired DNA damage may be particularly problematic.

An additional system that has emerged as a regulator of the DNA damage response is the body's circadian clock ${ }^{7}$, which governs many aspects of cellular, tissue, and organismal biochemistry and physiology with a periodicity of $24 \mathrm{hr}$. At the molecular level, the circadian clock is composed of a transcription-translation feedback loop in which the CLOCK-BMAL1 heterodimeric protein complex binds to E-box elements in promoters of target clock-control genes (CCGs) to modulate gene expression ${ }^{8}$. Two target CCGs include cryptochrome (CRY) and period (PER), which ultimately heterodimerize and feedback to inhibit CLOCK-BMAL1 activity. A secondary loop is governed by retinoic acid receptor orphan receptor (ROR) and REV-ERB proteins that bind in a competitive manner to ROR elements in the BMAL1 promoter to influence BMAL1 expression ${ }^{9}$. It has been estimated that up to $40-50 \%$ of all genes are regulated by the circadian clock in at least one tissue ${ }^{10}$, and thus it is expected that some of these gene products likely influence cisplatin DNA damage responses. For example, genes encoding the rate-limiting NER factor XPA (xeroderma pigmentosum group A) and anti-mitotic protein kinase Wee1 are well-recognized transcriptional targets of the circadian clock $^{11,12}$. Additional studies have found that the time of exposure 
during the $24 \mathrm{hr}$ day to cisplatin and other related DNA damaging agents like UV radiation impacts the efficiency of DNA repair and corresponding cell lethality ${ }^{13-17}$.

Genetic studies in cultured cells and mice have dominated experimental analyses of the circadian clock and its role in the pathophysiology of numerous disease states over the past 20 years ${ }^{7,18,19}$. However, recent drug discovery screens have identified compounds that bind to and regulate protein components of the circadian clock machinery ${ }^{20,21}$. These small molecules may therefore provide a new way to modulate cell and tissue biology under normal or pathological conditions and in response to external stressors, such as chemotherapy. The purpose of the current study was to examine whether circadian clock-modulating compounds can be used to alter the expression of genes relevant to cisplatin DNA damage responses and thus control the anti-proliferative effects of this common anti-cancer therapeutic drug in cultured cells in vitro.

\section{Materials And Methods}

\section{Cell culture and materials}

U2OS and HaCaT cells were maintained in DMEM (HyClone) containing an additional $2 \mathrm{mM} \mathrm{L-glutamine}$ (Gibco), 100 units $/ \mathrm{ml}$ penicillin, $100 \mu \mathrm{g} / \mathrm{ml}$ streptomycin, and 10\% fetal bovine serum. A549 cells were cultured in F-12K medium (Gibco) containing 10\% fetal bovine serum and antibiotics. All cells were grown in a humidified $37^{\circ} \mathrm{C}$ incubator with $5 \% \mathrm{CO}_{2}$. The clock modulating compounds $\mathrm{KS} 15$ (Glixx Laboratories) and SR8278 (Sigma) were prepared as 10 mM stocks in DMSO (Fisher Chemical). Cisplatin (Sigma) was prepared as a $3 \mathrm{mM}$ stock in PBS (HyClone). Drugs were used at the final concentrations described in the figure legends.

\section{Cell proliferation assays}

Cells were seeded in 96-well plates at a density of 3,000-5,000 cells per well in $100 \mu$ l of media and grown for 2-3 days until confluent. Cells were then treated for 48 hours in the presence or absence of the indicated concentrations of KS15, SR8278, and cisplatin. Media was then aspirated, and cells were incubated for either $4 \mathrm{hr}$ (U2OS) or $1 \mathrm{hr}$ (HaCaT, A549) with $100 \mu \mathrm{l}$ of culture medium containing 0.25 $\mathrm{mg} / \mathrm{ml}$ methyl-thiazolyl diphenyl-tetrazolium bromide (MTT). After aspiration of the culture media, MTT crystals were solubilized in $100 \mu$ of DMSO and absorbance was measured at $570 \mathrm{~nm}$ using a Synergy H1 spectrophotometer (Bio-Tek). GraphPad Prism (version 9.0) was used to normalize the absorbance values, perform non-linear regressions, and calculate $\mathrm{IC}_{50}$ values.

mRNA expression analyses

RNA was purified using an RNeasy Plus Micro Kit (Qiagen), quantified on a NanoDrop One spectrophotomer (ThermoFisher), and then reverse transcribed using a QuantiTect Reverse Transcription Kit (Qiagen). Quantitative PCR reactions were prepared using 2X TaqMan Fast Universal PCR Master Mix (ThermoFisher) and TaqMan probes targeting XPA (Hs00902270), Wee1 (Hs01119384), and beta-2- 
microglobulin (B2M) (Hs0187842) (Applied Biosystems). DNA was amplified on a CFX96 Real-Time PCR Detection System (Bio-Rad) using an initial 3 min melting step at $95^{\circ} \mathrm{C}$ followed by 40 cycles of $95^{\circ} \mathrm{C}$ for $10 \mathrm{~s}$ and $55^{\circ} \mathrm{C}$ for $30 \mathrm{~s}$. The $\Delta \Delta \mathrm{C}_{\mathrm{t}}$ method was used to calculate fold changes in gene expression using $\mathrm{B} 2 \mathrm{M}$ as an internal housekeeping gene.

\section{Protein immunoblotting}

Cells were lysed in cold Triton X-100 lysis buffer (20 mM Tris-HCl, pH 7.5, 150 mM NaCl, 1 mM EDTA, 1 mM EGTA, and 1\% Triton X-100) and incubated on ice for 15-20 min with occasional vortexing. Soluble lysates were obtained by centrifugation at maximum speed in a microcentrifuge. Equal amounts of protein were separated on $8 \%$ Tris-Glycine SDS gels. Proteins were transferred to a nitrocellulose membrane using a semi-dry transfer apparatus and stained with $0.5 \%$ Ponceau S (Sigma) to ensure equal loading and transfer. The blots were blocked in in 5\% non-fat milk in TBST (Tris-buffered saline containing $0.1 \%$ Tween-20) and then probed overnight with primary antibodies against XPA (1:1000 dilution; sc-28353), Wee1 (1:1000; sc-5285), or p21 (1:1000; sc-2646), which were obtained from Santa Cruz Biotechnology. After washing with TBST, blots were probed with HRP-coupled anti-mouse or antirabbit IgG (Invitrogen) secondary antibodies for one hour at room temperature. Chemiluminescence was visualized with Clarity Western ECL substrate (Bio-Rad) or SuperSignal West Femto substrate (Thermo Scientific) using a Molecular Imager Chemi-Doc XRS+ imaging system (Bio-Rad). Image Lab (Bio-Rad) was used to quantify signal intensities, which were normalized to the Ponceau S stain.

\section{DNA immunoblotting}

Genomic DNA was purified using a GenElute Mammalian Genomic DNA Miniprep Kit (Sigma) and quantified using either a NanoDrop One spectrophotometer or Qubit 4.0 fluorometer (Thermo Fisher). Equal amounts of genomic DNA (50-100 ng) were boiled for $10 \mathrm{~min}$, neutralized on ice with an equal volume of $2 \mathrm{M}$ cold ammonium acetate $(\mathrm{pH} \mathrm{7.0)}$ ) and then loaded onto a nitrocellulose membrane using a 96-well dot blot apparatus. After drying for $30 \mathrm{~min}$ at $80^{\circ} \mathrm{C}$, blots were blocked in milk and probed with anti-cisplatin-modified DNA antibody (1:10000 dilution; Abcam ab103261) and then HRP-coupled anti-rat IgG secondary antibody. Chemiluminescence was detected and quantified as described for protein immunoblotting. In some cases, blots were stained with SYBR Gold stain (1:10000; Invitrogen).

\section{Flow cytometry}

Cells were harvested by trypsinization and centrifuged at 1,500 rpm for 5 minutes. After washing with PBS, cells were fixed overnight with $70 \%$ ice-cold ethanol at $-20^{\circ} \mathrm{C}$. Fixed cells were washed with PBS, centrifuged at 4,000 rpm for 5 minutes, and resuspended in PBS containing $10 \mu \mathrm{g} / \mathrm{ml}$ RNase A and 50 $\mu \mathrm{g} / \mathrm{ml}$ propidium iodide. Cells were analyzed for DNA content using an Accuri C6 flow cytometer. Cell cycle distribution was determined after appropriate gating of cell populations. 
All experiments were performed at least 3 times, and samples sizes are indicated in the figure legends. Differences between treatment groups were evaluated using GraphPad Prism (version 9.0) and either ttests or one-way ANOVAs. Data were considered statistically significant at p-values less than 0.05 . In most graphs, average and SEM are displayed along with values for individual experimental samples.

\section{Data availability}

All data generated or analysed during this study are included in this published article.

\section{Results}

\section{The CRY inhibitor KS15 and REV-ERB antagonist SR8278 promote cell proliferation in cisplatin-treated cells}

A schematic of the circadian clock transcription-translation feedback system is provided in Figure $1 \mathrm{~A}$ along with the two clock-modulating compounds (KS15 and SR8278) that were tested in this study due to their documented ability to alter circadian promoter activity and/or CCG expression ${ }^{22-24}$. CRY represses CLOCK-BMAL1 transcriptional activity, and the CRY inhibitor KS15 has been shown increase in the expression of CCGs such as Wee1 in cells in vitro ${ }^{22,23}$. REV-ERB, which negatively regulates BMAL1 expression by binding to its promoter, can be antagonized with SR8278 ${ }^{24}$. Because the effectiveness of the anti-cancer drug cisplatin is impacted by cellular processes that are regulated by the circadian clock, such as DNA repair and cell cycle checkpoints ${ }^{18,25}$, the effect of KS15 and SR8278 on DNA repair, cell cycle progression, and cell viability were tested both alone and in various combinations.

U2OS cells selected for this initial analysis because this cell line expresses circadian proteins and maintains circadian rhythmicity upon stimulation ${ }^{26}$. As shown in Figure 1B, treatment of asynchronously growing U2OS cells with increasing concentrations of KS15 and SR8278 (10, 20, and $50 \mu \mathrm{M})$ did not dramatically influence cell proliferation either alone or in combination with a fixed concentration $(10 \mu \mathrm{M})$ of the other compound. Cells were then co-treated with the clock drugs in combination with increasing concentrations of cisplatin. Interestingly, treatment of U20S cells with $50 \mu \mathrm{M} \mathrm{KS} 15$ partially protected cells against the anti-proliferative effects of cisplatin (Figure 1C). Similar results were observed with 50 $\mu \mathrm{M}$ SR8278 (Figure 1D). Additional cell proliferation assays were carried out with various concentrations of KS15 and SR8278 alone and in combination, and IC ${ }_{50}$ values for cisplatin were calculated with the different treatment combinations. As shown in Table 1, the highest concentrations of KS15 and SR8278 led to statistically significant changes in $\mathrm{IC}_{50}$ values, which increased from $18 \mu \mathrm{M}$ in vehicle-treated cells to approximately $40 \mu \mathrm{M}$ in cells treated with the clock modulating compounds. Similar levels of protection were also observed in cells treated with a combination of lower concentrations of KS15 and SR8278.

To confirm these results in additional cell lines, $\mathrm{HaCaT}$ keratinocytes, which also have a functional circadian clock ${ }^{27}$, were treated with KS15 alone and in combination with SR8278. As shown in Figure 2A, 
the compounds alone had no significant effect on cell proliferation. However, when HaCaT cells were treated with $50 \mu \mathrm{M} \mathrm{KS15}$ in combination with $10 \mu \mathrm{M}$ SR8278 in the presence of increasing concentrations of cisplatin, they displayed significantly higher levels of cell proliferation than vehicle-treated cells (Figure 2B, Table 1). Experiments were then repeated in A549 lung carcinoma cells, which displayed little sensitivity to the clock drugs alone (Figure $2 \mathrm{C}$ ) but were protected against cisplatin by co-treatment with KS15 and SR8278 (Figure 2D, Table 1). Thus, we conclude from these studies that the clock modulating compounds KS15 and SR8278 can be used to counteract the anti-proliferative effects of the cancer chemotherapy drug cisplatin in cultured human cells in vitro.

\section{KS15 and REV-ERB treatment increases the expression of the CCGs XPA and Wee1}

Cellular sensitivity to cisplatin is impacted by many factors, including DNA repair and cell cycle phase ${ }^{3,28,29}$. Because the NER factor XPA is regulated at the transcriptional level by the circadian clock machinery ${ }^{11,14}$, XPA mRNA expression was measured by RT-qPCR in U2OS treated with KS15 and SR278 alone and in combination. As shown in Figure 3A, treatment with the combination of KS15 and SR8278 increased XPA expression by approximately 2.9-fold. Expression of Wee1, another well-known target of the circadian cloc $^{12}$, was also increased by KS15+SR8278 by 3.7-fold (Figure 3B). To determine if the increased mRNA levels are correlated with changes at the protein level, western blotting was performed using cell lysates from U2OS cells treated with the clock compounds. As shown in Figure 3C, D, protein levels of both XPA and Wee1 were increased by nearly 2-fold in cells treated with the combination of KS15 and SR8278.

\section{KS15 and REV-ERB promote cisplatin-DNA adduct removal}

XPA is a rate-limiting factor in NER ${ }^{30}$, and thus the increased expression of XPA observed in Figure 3 may facilitate the removal of cisplatin-induced intra-strand DNA adducts. U2OS cells were treated with vehicle or KS15 and SR8278 in the presence of increasing concentrations of cisplatin, and then genomic DNA was purified for analysis by DNA immunoblotting with an anti-cisplatin-DNA adduct antibody. As shown in Figure 4A, cisplatin treatment for $2 \mathrm{hr}$ induced a dose-dependent induction of adduct formation. Cell culture media was then replaced with fresh medium containing the clock drugs but lacking cisplatin to determine how the clock drugs impact the repair of these DNA adducts after additional $22 \mathrm{hr}$ of incubation. Consistent with XPA's role in promoting NER and its up-regulation by treatment of cells with KS15+SR8278, fewer remaining cisplatin-DNA adducts were observed in the cells treated with KS15 and SR8278 at the $24 \mathrm{hr}$ time point than in the vehicle-treated cells (Figure 4A, B).

\section{KS15 and REV-ERB promote G1 cell cycle arrest}

Because the Wee1 kinase prevents the entry of cells into mitosis, the increased expression of Wee1 observed in Figure 3 may be expected to lead to an enrichment of cells in the G2 phase of the cell cycle. However, when U2OS cells were treated with KS15 and SR8278 (alone and in combination) and analyzed for DNA content by flow cytometry, a modest reduction in cells in late $S / 2$ phase was observed (Figure 5A). Rather, quantitation of cell cycle distribution from 3 independent experiments showed there to a 
modest but statistically significant increase in cells in the G1 phase of the cell cycle (Figure 5B) when cells were treated with the combination of KS15 and SR8278. To identify a potential mechanism for this

increased fraction of cells in $\mathrm{G} 1$ phase, expression of the cyclin-dependent kinase inhibitory protein $\mathrm{p} 21^{31}$, which was previously reported to be a target of the circadian clock machinery ${ }^{32}$, was examined. Western blotting showed that $\mathrm{p} 21$ protein levels were increased by treatment of SR8278 alone or in combination with KS15 (Figure 5C). Thus, cell cycle progression is impacted by treatment with small molecules that target circadian clock proteins in a similar manner as for DNA repair.

\section{Discussion}

Though there is growing interest in using small molecule drugs that target the circadian clock to ameliorate the pathophysiology of metabolic disorder and cancer ${ }^{20,21}$, little work has been done to study how these compounds can be used to modulate responses to DNA damage caused by cisplatin and other related agents. The current study is the first to analyze how the CRY inhibitor KS15 and REV-ERB antagonist SR8278 affect the anti-proliferative effects of cisplatin. Because CRY and REV-ERB function to suppress CLOCK-BMAL1 expression and/or activity ${ }^{8}$, pharmacological inhibition of CRY and REV-ERB is expected to de-repress the transcription complex and promote the expression of CCGs that may be relevant for cellular responses to cisplatin. Consistent with this hypothesis, the NER factor XPA and cell cycle regulators Wee1 and p21 were found here to be increased by treatment with KS15 and SR8278 (Figures 3,5$)$. This increased gene expression was correlated with more efficient NER (Figure 4), a higher fraction of cells in $\mathrm{G} 1$ phase of the cell cycle (Figure 5), and resistance to the anti-proliferative effects of cisplatin (Figures 1, 2 and Table 1).

The work here focused on a limited set of gene products relevant to cisplatin responses, and thus it is possible that additional genes and signaling pathways also contribute to the effects of KS15 and SR8278 on cellular responses to cisplatin. Thus, additional work may be needed to fully understand the effects of these compounds and to determine whether the effects are dependent on the circadian clock machinery. Moreover, as methods for synchronizing the circadian behavior of the cultured cells, such as high serum concentrations or dexamethasone, were not used in this work, it is possible that the effectiveness of KS15 and SR8278 in protecting against cisplatin may be altered by the timing of treatment during normal circadian rhythms. Such approaches in cultured cells in vitro and in animal models in vivo are therefore warranted to advance the idea of using clock modulating compounds to alter cellular responses to DNA damage.

Based on the results of this study, the use of KS15 and SR8278 may be relevant to limit the toxicity caused by cisplatin in normal body tissues such as the kidney and brain, which are known to be impacted by cisplatin chemotherapy regimens. However, these compounds may also limit the effectiveness of cisplatin towards cancer cells. Thus, targeted drug delivery could be necessary to optimize desired therapeutic outcomes. Nonetheless, while the work here focused on compounds that target circadian clock components that are expected to enhance CLOCK-BMAL1 transcriptional output, there are other 
clock-modulating compounds ${ }^{21}$, including the CRY stabilizer KL001 ${ }^{33}$ and REV-ERB agonist SR9009 ${ }^{34}$, that in principle may sensitize cells to cisplatin by inhibiting the expression of CCGs important in DNA repair and cisplatin responses. Future studies should therefore explore these additional classes of drugs in cisplatin treatment.

\section{Declarations}

\section{Acknowledgements}

The authors thank the WSU Proteome Analysis Laboratory for the use of equipment to carry out this work.

\section{Author Contributions}

MGK designed and supervised the study. NA, BC, and MGK performed experiments. MGK wrote the initial draft of the manuscript, and the final version was approved by all authors.

\section{Funding}

This work was supported by start-up funding provided by Wright State University and by a grant from Ohio Cancer Research (\#5020).

\section{Competing financial interest}

The authors declare that they have no conflicts of interest.

\section{References}

1. Jamieson, E. R. \& Lippard, S. J. Structure, Recognition, and Processing of Cisplatin-DNA Adducts. Chem. Rev. 99, 2467-2498 (1999).

2. Cohen, S. M. \& Lippard, S. J. Cisplatin: from DNA damage to cancer chemotherapy. Prog. Nucleic Acid Res. Mol. Biol. 67, 93-130 (2001).

3. Zamble, D. B., Mu, D., Reardon, J. T., Sancar, A. \& Lippard, S. J. Repair of cisplatin-DNA adducts by the mammalian excision nuclease. Biochemistry 35, 10004-10013 (1996).

4. Sancar, A. Mechanisms of DNA Repair by Photolyase and Excision Nuclease (Nobel Lecture). Angew. Chemie (International ed.in English) 55, 8502-8527 (2016).

5. Sancar, A., Lindsey-Boltz, L. A., Unsal-Kacmaz, K. \& Linn, S. Molecular mechanisms of mammalian DNA repair and the DNA damage checkpoints. Annu. Rev. Biochem. 73, 39-85 (2004).

6. Ciccia, A. \& Elledge, S. J. The DNA damage response: making it safe to play with knives. Mol. Cell 40, 179-204 (2010). 
7. Mohawk, J. A., Green, C. B. \& Takahashi, J. S. Central and peripheral circadian clocks in mammals. Annu. Rev. Neurosci. 35, 445-462 (2012).

8. Takahashi, J. S. Transcriptional architecture of the mammalian circadian clock. Nat. Rev. 18, 164179 (2017).

9. Cho, H. et al. Regulation of circadian behaviour and metabolism by REV-ERB-a and REV-ERB- $\beta$. Nature 485, 123-127 (2012).

10. Zhang, R., Lahens, N. F., Ballance, H. I., Hughes, M. E. \& Hogenesch, J. B. A circadian gene expression atlas in mammals: implications for biology and medicine. Proc. Natl. Acad. Sci. U. S. A. 111, 1621916224 (2014).

11. Kang, T. H., Reardon, J. T., Kemp, M. \& Sancar, A. Circadian oscillation of nucleotide excision repair in mammalian brain. Proc. Natl. Acad. Sci. U. S. A. 106, 2864-2867 (2009).

12. Matsuo, T. et al. Control mechanism of the circadian clock for timing of cell division in vivo. Science 302, 255-259 (2003).

13. Gaddameedhi, S., Selby, C. P., Kaufmann, W. K., Smart, R. C. \& Sancar, A. Control of skin cancer by the circadian rhythm. Proc. Natl. Acad. Sci. U. S. A. 108, 18790-18795 (2011).

14. Kang, T. H., Lindsey-Boltz, L. A., Reardon, J. T. \& Sancar, A. Circadian control of XPA and excision repair of cisplatin-DNA damage by cryptochrome and HERC2 ubiquitin ligase. Proc. Natl. Acad. Sci. U. S. A. 107, 4890-4895 (2010).

15. Gaddameedhi, S., Selby, C. P., Kemp, M. G., Ye, R. \& Sancar, A. The circadian clock controls sunburn apoptosis and erythema in mouse skin. J. Invest. Dermatol. 135, 1119-1127 (2015).

16. Yang, Y. et al. Cisplatin-DNA adduct repair of transcribed genes is controlled by two circadian programs in mouse tissues. Proc. Natl. Acad. Sci. U. S. A. 115, E4777-E4785 (2018).

17. Dakup, P. P. et al. The circadian clock regulates cisplatin-induced toxicity and tumor regression in melanoma mouse and human models. Oncotarget 9, 14524-14538 (2018).

18. Antoch, M. P., Kondratov, R. V \& Takahashi, J. S. Circadian clock genes as modulators of sensitivity to genotoxic stress. Cell Cycle 4, 901-907 (2005).

19. Sancar, A. et al. Circadian clock control of the cellular response to DNA damage. FEBS Lett. 584, 2618-2625 (2010).

20. Gloston, G. F., Yoo, S. H. \& Chen, Z. J. Clock-Enhancing Small Molecules and Potential Applications in Chronic Diseases and Aging. Front. Neurol. 8, 100 (2017).

21. Chen, Z., Yoo, S. H. \& Takahashi, J. S. Development and Therapeutic Potential of Small-Molecule Modulators of Circadian Systems. Annu. Rev. Pharmacol. Toxicol. 58, 231-252 (2018).

22. Chun, S. K. et al. A synthetic cryptochrome inhibitor induces anti-proliferative effects and increases chemosensitivity in human breast cancer cells. Biochem. Biophys. Res. Commun. 467, 441-446 (2015).

23. Jang, J. et al. The cryptochrome inhibitor KS15 enhances E-box-mediated transcription by disrupting the feedback action of a circadian transcription-repressor complex. Life Sci. 200, 49-55 (2018). 
24. Kojetin, D., Wang, Y., Kamenecka, T. M. \& Burris, T. P. Identification of SR8278, a synthetic antagonist of the nuclear heme receptor REV-ERB. ACS Chem. Biol. 6, 131-134 (2011).

25. Sancar, A. et al. Circadian clock, cancer, and chemotherapy. Biochemistry 54, 110-123 (2015).

26. Vollmers, C., Panda, S. \& DiTacchio, L. A high-throughput assay for siRNA-based circadian screens in human U2OS cells. PLoS One 3, e3457 (2008).

27. Spörl, F. et al. A circadian clock in HaCaT keratinocytes. J. Invest. Dermatol. 131, 338-348 (2011).

28. Evans, D. L., Tilby, M. \& Dive, C. Differential sensitivity to the induction of apoptosis by cisplatin in proliferating and quiescent immature rat thymocytes is independent of the levels of drug accumulation and DNA adduct formation. Cancer Res. 54, 1596-1603 (1994).

29. Hutcherson, R. J. \& Kemp, M. G. ATR kinase inhibition sensitizes quiescent human cells to the lethal effects of cisplatin but increases mutagenesis. Mutat. Res. 816-818, 111678 (2019).

30. Kang, T. H., Reardon, J. T. \& Sancar, A. Regulation of nucleotide excision repair activity by transcriptional and post-transcriptional control of the XPA protein. Nucleic Acids Res. 39, 3176-3187 (2011).

31. El-Deiry, W. S. p21(WAF1) Mediates Cell-Cycle Inhibition, Relevant to Cancer Suppression and Therapy. Cancer Res. 76, 5189-5191 (2016).

32. Gréchez-Cassiau, A., Rayet, B., Guillaumond, F., Teboul, M. \& Delaunay, F. The circadian clock component BMAL1 is a critical regulator of $\mathrm{p} 21 \mathrm{WAF} 1 / \mathrm{CIP} 1$ expression and hepatocyte proliferation. J. Biol. Chem. 283, 4535-4542 (2008).

33. Lee, J. W. et al. Development of Small-Molecule Cryptochrome Stabilizer Derivatives as Modulators of the Circadian Clock. ChemMedChem 10, 1489-1497 (2015).

34. Solt, L. A. et al. Regulation of circadian behaviour and metabolism by synthetic REV-ERB agonists. Nature 485, 62-68 (2012).

\section{Table}

Table 1. Summary of $I_{50}$ values for cisplatin-treated human cells. Non-linear regression was used to calculate $\mathrm{IC}_{50}$ values for U2OS, $\mathrm{HaCaT}$, and A549 cells treated with cisplatin and the indicated concentrations of KS15 and SR8278 as shown in Figures 1 and 2. One-way ANOVAs were used to identify KS15 and SR8278 treatment conditions that significantly impacted $I_{50}$ values in comparison to the vehicle (DMSO)-treated control. 


\begin{tabular}{|c|c|c|c|c|}
\hline Cell Line & Treatment & Average $\mathrm{IC}_{50}$ & SEM & $\mathrm{p}$-value \\
\hline \multirow[t]{12}{*}{ U2OS } & DMSO & 18.4 & 2.15 & - \\
\hline & $10 \mu \mathrm{M}$ KS15 & 25.6 & 2.01 & 0.811 \\
\hline & $20 \mu \mathrm{M}$ KS15 & 29.9 & 3.50 & 0.290 \\
\hline & $50 \mu \mathrm{M}$ KS15 & 41.9 & 4.11 & 0.001 \\
\hline & $10 \mu \mathrm{M}$ SR8278 & 25.1 & 3.19 & 0.864 \\
\hline & $20 \mu \mathrm{M}$ SR8178 & 25.8 & 3.75 & 0.781 \\
\hline & $50 \mu \mathrm{M}$ SR8178 & 37.1 & 6.11 & 0.014 \\
\hline & $10 \mu \mathrm{M} \mathrm{SR}+10 \mu \mathrm{M} \mathrm{KS}$ & 33.3 & 3.72 & 0.082 \\
\hline & $10 \mu \mathrm{M} \mathrm{SR}+20 \mu \mathrm{M}$ KS & 35.5 & 3.99 & 0.031 \\
\hline & $10 \mu \mathrm{M} \mathrm{SR}+50 \mu \mathrm{M}$ KS & 40.8 & 3.72 & 0.002 \\
\hline & $10 \mu \mathrm{M} \mathrm{KS}+20 \mu \mathrm{M}$ SR & 36.7 & 5.29 & 0.018 \\
\hline & $10 \mu \mathrm{M} \mathrm{KS}+50 \mu \mathrm{M}$ SR & 41.3 & 4.94 & 0.001 \\
\hline \multirow[t]{8}{*}{ НаCaT } & DMSO & 3.2 & 0.18 & - \\
\hline & $10 \mu \mathrm{M} \mathrm{KS} 15$ & 4.9 & 0.56 & 0.711 \\
\hline & $20 \mu \mathrm{M}$ KS15 & 4.9 & 0.85 & 0.723 \\
\hline & $50 \mu \mathrm{M}$ KS15 & 6.0 & 1.21 & 0.237 \\
\hline & $10 \mu \mathrm{M}$ SR8278 & 4.5 & 0.49 & 0.865 \\
\hline & $10 \mu \mathrm{M} \mathrm{SR}+10 \mu \mathrm{M}$ KS & 5.8 & 1.07 & 0.306 \\
\hline & $10 \mu \mathrm{M} \mathrm{SR}+20 \mu \mathrm{M}$ KS & 6.6 & 1.44 & 0.118 \\
\hline & $10 \mu \mathrm{M} \mathrm{SR}+50 \mu \mathrm{M}$ KS & 7.4 & 1.34 & 0.042 \\
\hline \multirow[t]{8}{*}{ A549 } & DMSO & 9.4 & 1.55 & - \\
\hline & $10 \mu \mathrm{M}$ KS15 & 9.9 & 1.84 & 0.999 \\
\hline & $20 \mu \mathrm{M}$ KS15 & 12.5 & 1.06 & 0.661 \\
\hline & $50 \mu \mathrm{M}$ KS15 & 12.8 & 2.39 & 0.574 \\
\hline & $10 \mu \mathrm{M}$ SR8278 & 8.6 & 1.04 & 0.999 \\
\hline & $10 \mu \mathrm{M} \mathrm{SR}+10 \mu \mathrm{M}$ KS & 12.6 & 0.91 & 0.633 \\
\hline & $10 \mu \mathrm{M} \mathrm{SR}+20 \mu \mathrm{M} \mathrm{KS}$ & 15.3 & 2.52 & 0.121 \\
\hline & $10 \mu \mathrm{M} S R+50 \mu \mathrm{M}$ KS & 17.3 & 1.39 & 0.023 \\
\hline
\end{tabular}


Figures

a

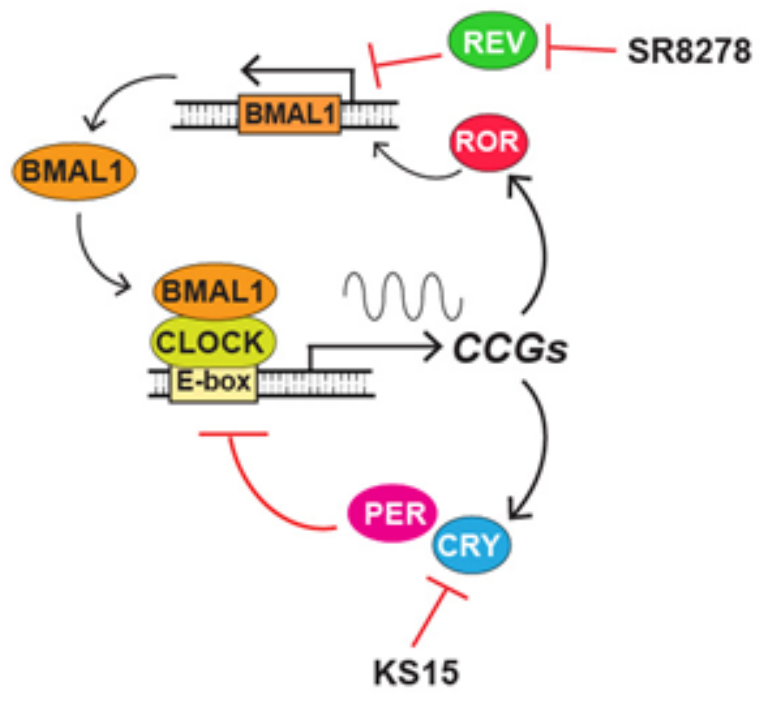

C

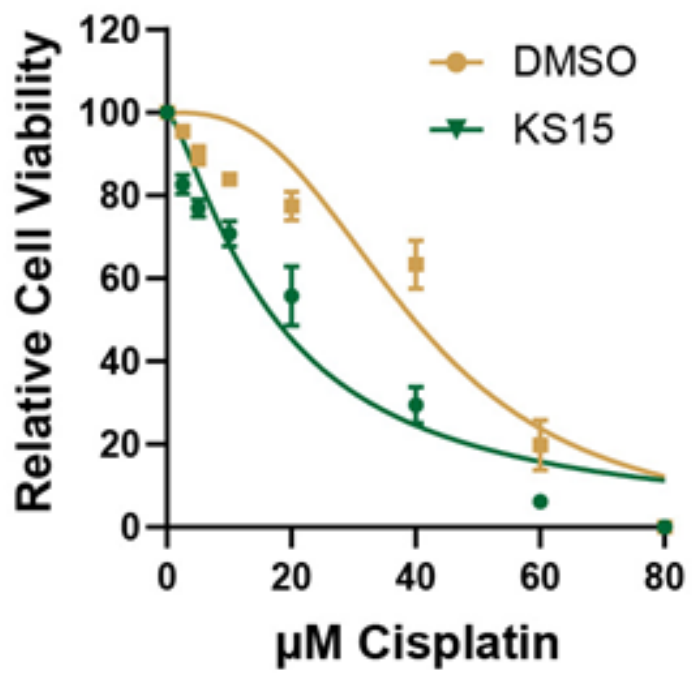

b
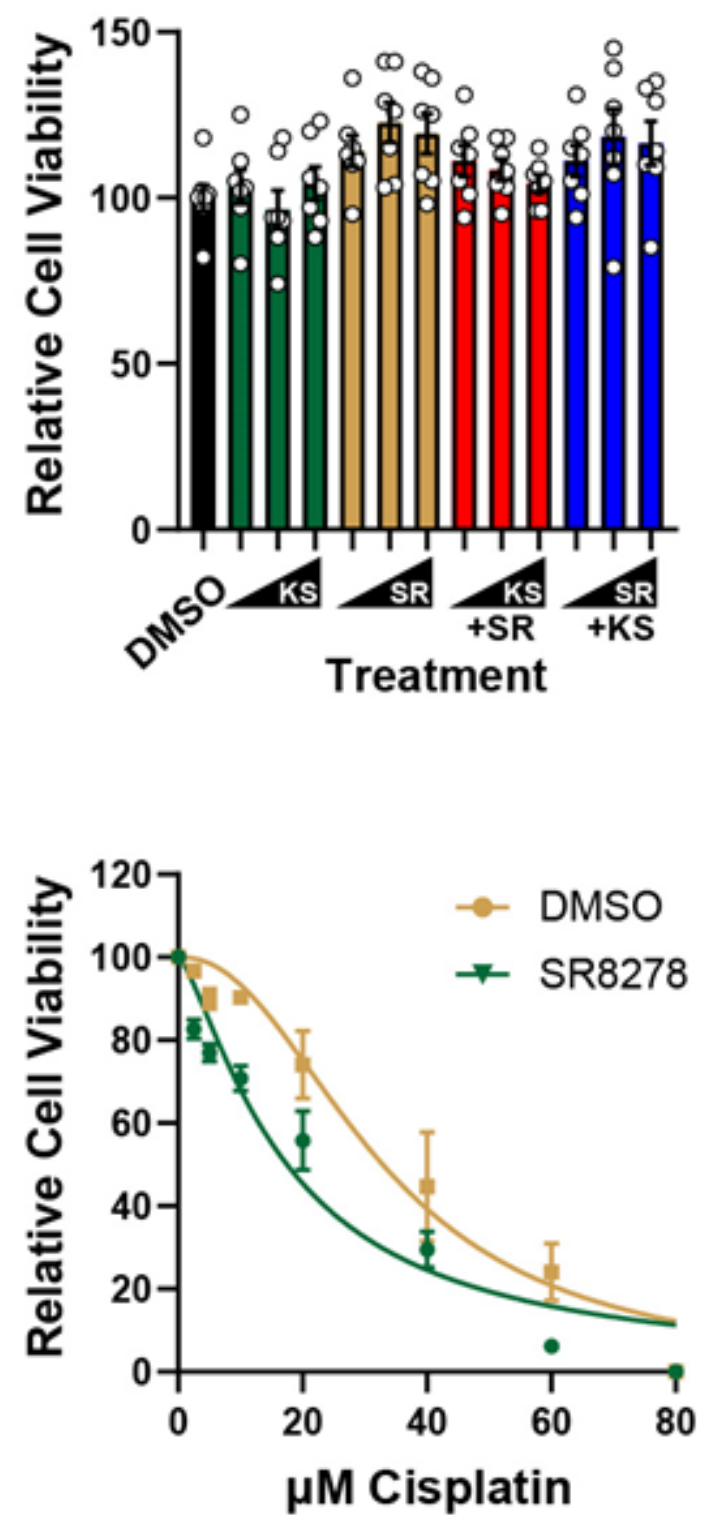

Figure 1

The cryptochrome inhibitor KS15 and REV-ERB antagonist SR8278 protect U2OS cells against cisplatin treatment. (a) Schematic of the circadian clock transcription-translation feedback loop. The CLOCKBMAL1 complex binds E-boxes in the promoters of target clock-control genes (CCGs), including the period (PER) and cryptochrome (CRY) that feedback to inhibit CLOCK-BMAL1 activity. Expression of BMAL1 is regulated by the retinoic acid receptor-related orphan receptor (ROR) and REV-ERB (REV) proteins that competitively bind to ROR elements (RORE) in the BMAL1 promoter. The CRY inhibitor KS15 and REV-ERB antagonist SR8278 have been shown to impact target gene and promoter activities. (b) U2OS cells were treated for 2 days with vehicle (0.1\% DMSO) or increasing concentrations $(10,20,50 \mu \mathrm{M})$ of KS15 (KS) and SR8278 (SR) in the absence or presence of $10 \mu \mathrm{M}$ of the other compound. MTT assays were 
performed to measure relative cell viability (average and SEM, $n=7$ ). (c) U2OS cells were treated with DMSO or $50 \mu \mathrm{M} \mathrm{KS15}$ and the indicated concentration of cisplatin for 2 days, and then MTT assays were performed to measure relative cell proliferation (average and SEM; $n=6$ ). (d) U2OS cells were treated as in (C) except with $50 \mu \mathrm{M}$ SR8278.
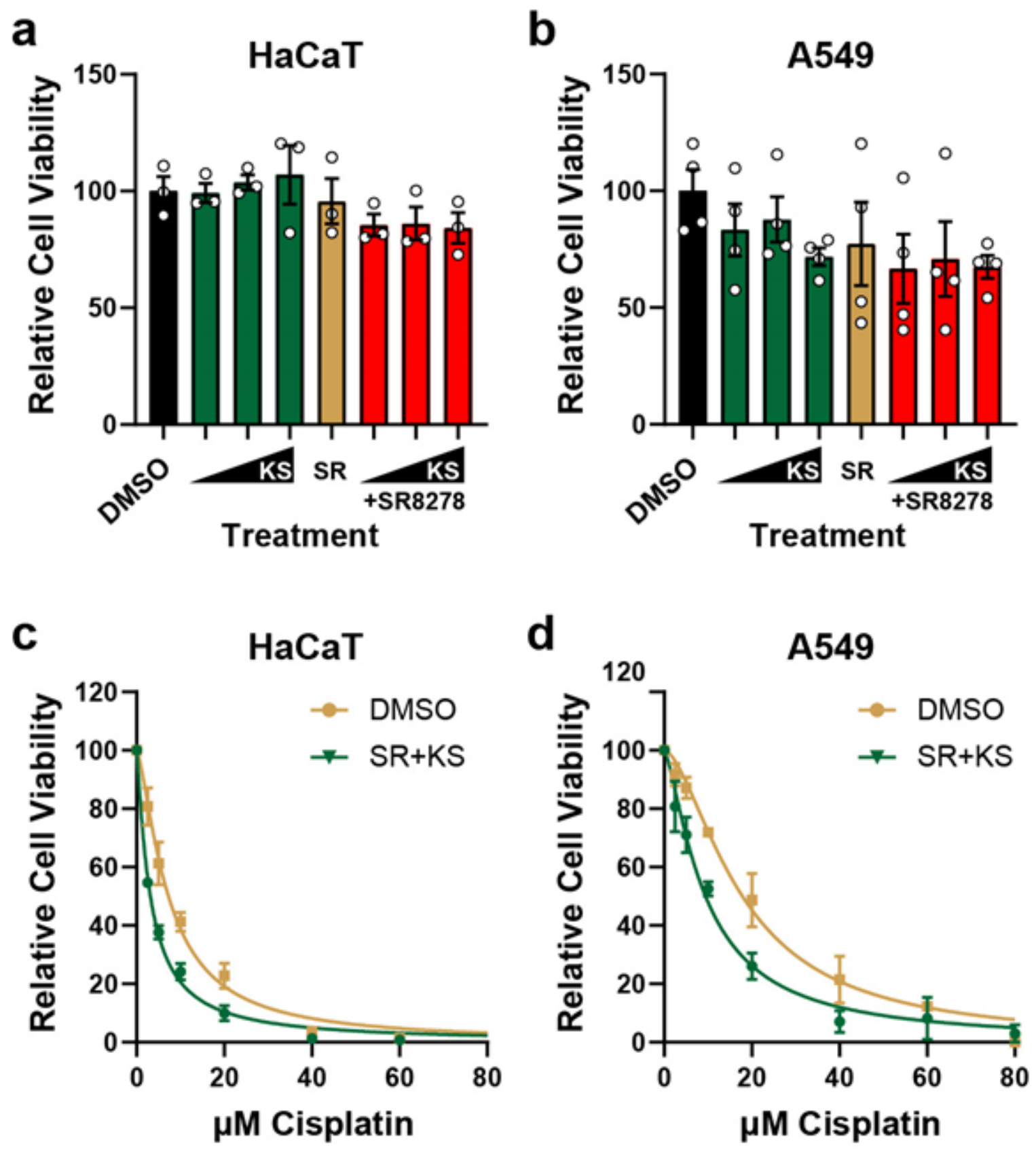

Figure 2

KS15 and SR8278 protect HaCaT and A549 cells against cisplatin treatment. (a) MTT assays were performed in HaCaT cells treated with KS15 and SR8278 (average and SEM, n=3). (b) HaCaT cells were treated with DMSO or a combination of $50 \mu \mathrm{M} \mathrm{KS15}$ and $10 \mu \mathrm{M}$ SR8278 along with the indicated concentrations of cisplatin. MTT assays were performed two days later (average and SEM, n=3). (c) A549 cells were treated as in (a), and MTT assays were performed (average and SEM, $n=4$ ). (d) MTT assays 
were performed in A549 cells were treated with cisplatin and DMSO or the combination of $50 \mu \mathrm{M} \mathrm{K15}$ and $10 \mu \mathrm{M}$ SR8278 (average and SEM, $\mathrm{n}=4$ ).

a

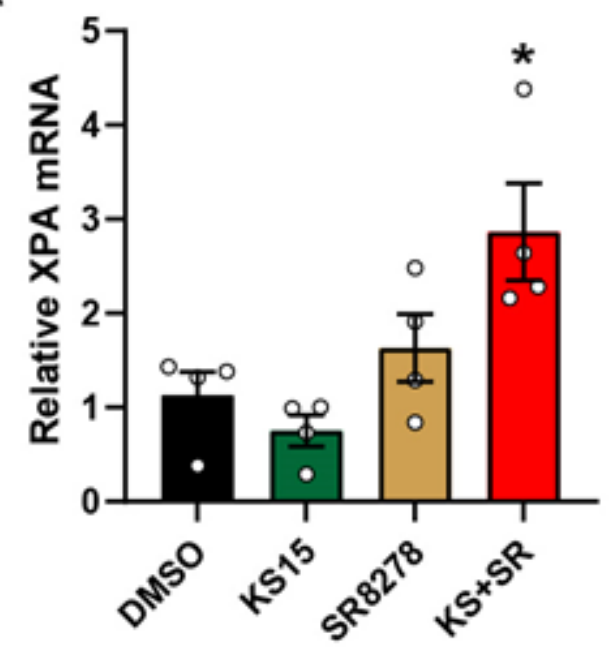

Treatment

C

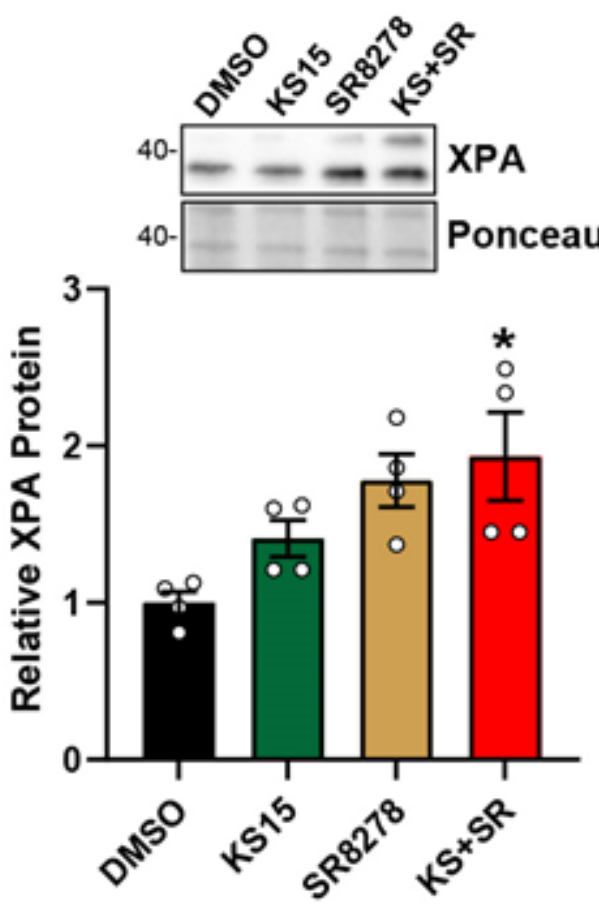

Treatment b

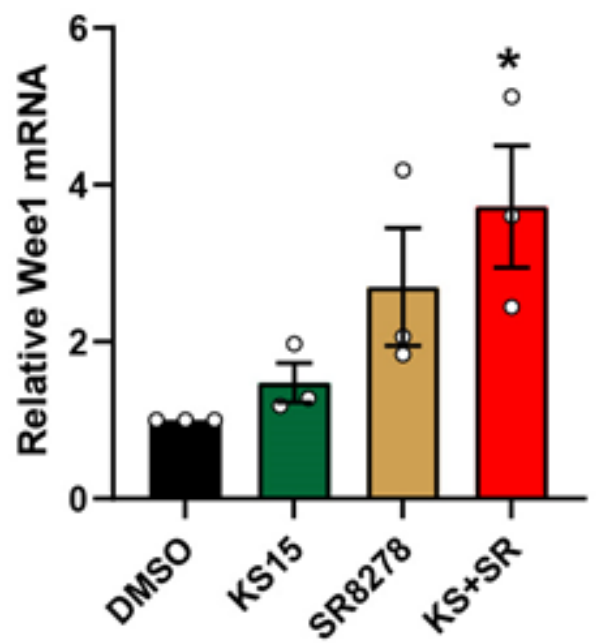

Treatment

d

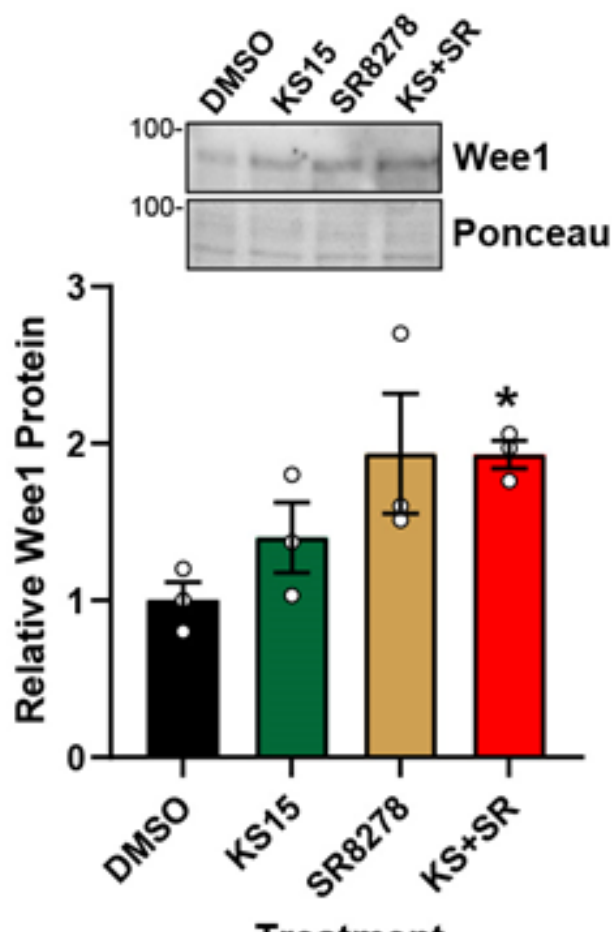

Treatment

Figure 3

KS15 and SR8278 treatment increase XPA and Wee1 expression in U2OS cells. (a) U2OS cells were treated with DMSO vehicle, $20 \mu \mathrm{M} \mathrm{KS15,} 10 \mu \mathrm{M}$ SR8278, or the combination of KS15 and SR8278 (KS+SR) for $24 \mathrm{hr}$. RNA was purified and analyzed for XPA mRNA expression by RT-qPCR (average and SEM, n=4). (b) Wee1 mRNA was analyzed as in (a). (c) Cell lysates from cells treated as in (a) were analyzed for XPA protein by immunoblotting (average and SEM, $n=4$ ). (d) Wee1 protein expression was 
analyzed as in (c). The asterisks indicate differences in mRNA protein expression in comparison to DMSO-treated control cells ( $p<0.05$; one-way ANOVA).

a

Cisplatin-

DNA Adduct Sybr Gold

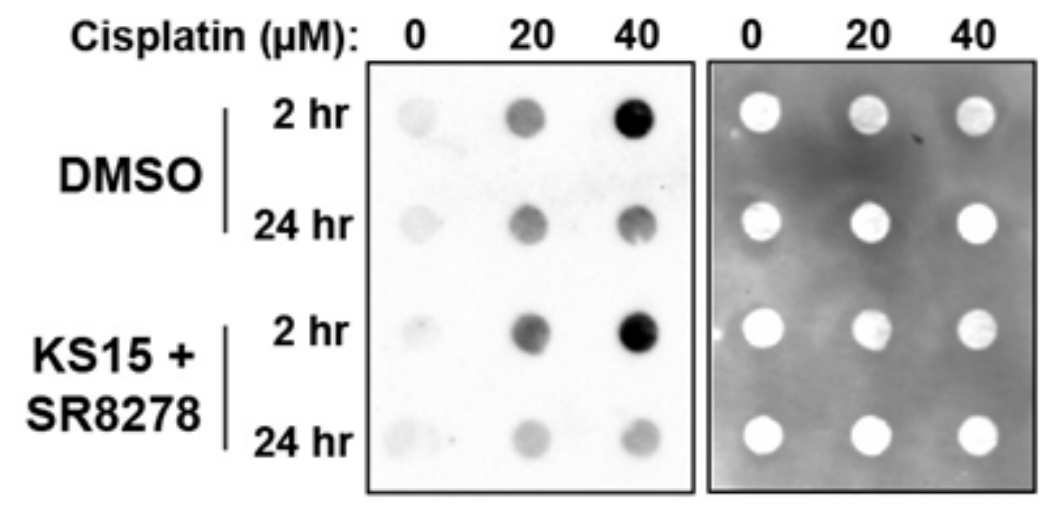

b

$\square$ DMSO

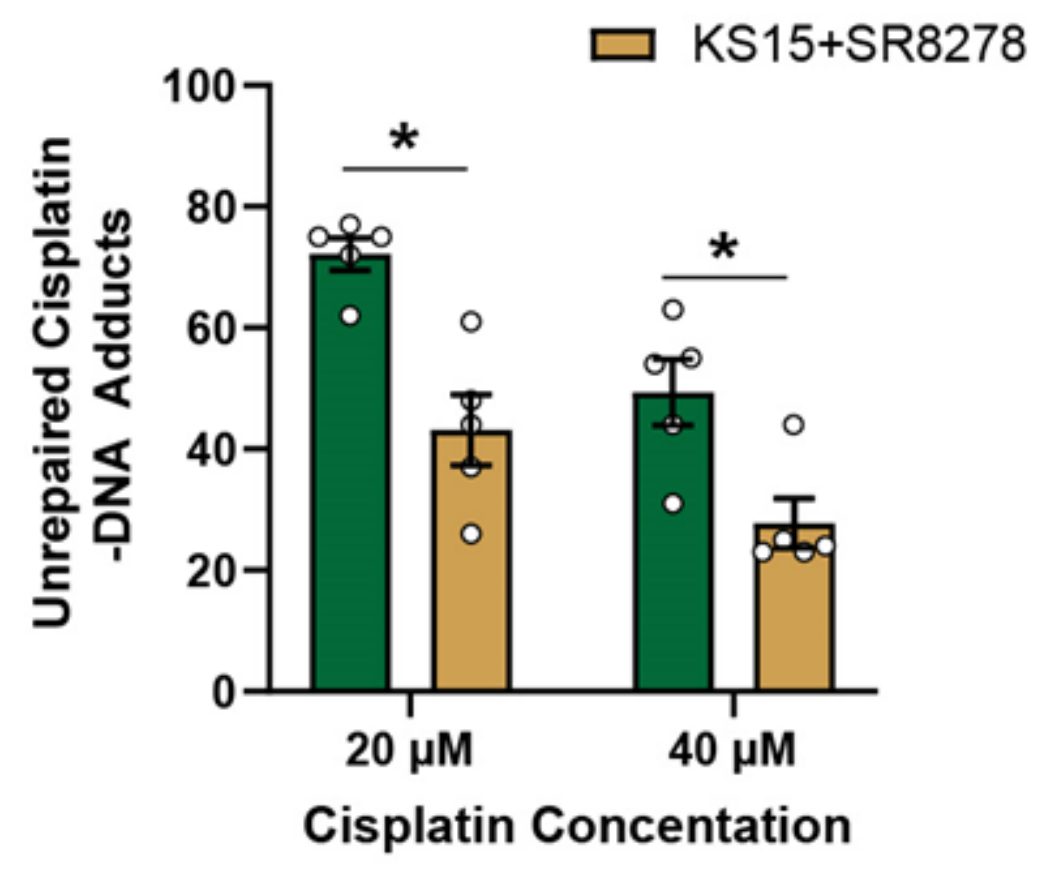

Figure 4

Treatment of cells with KS15 and SR8278 treatment promotes the removal of cisplatin-DNA adducts from

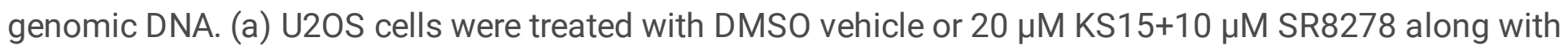
the indicated concentrations of cisplatin. Genomic DNA was purified from the cells 2 and $24 \mathrm{hr}$ later and analyzed by DNA immunoblotting with an anti-cisplatin DNA adduct antibody. Blots were then stained with SybrGold to visualize total DNA. (b) Quantitation of relative cisplatin-DNA adduct signal (average and SEM) at $24 \mathrm{hr}$ in relative to the $2 \mathrm{hr}$ time point $(n=5)$. The asterisks indicate significant differences in unrepaired cisplatin adducts (t-test, $p<0.05)$. 
a

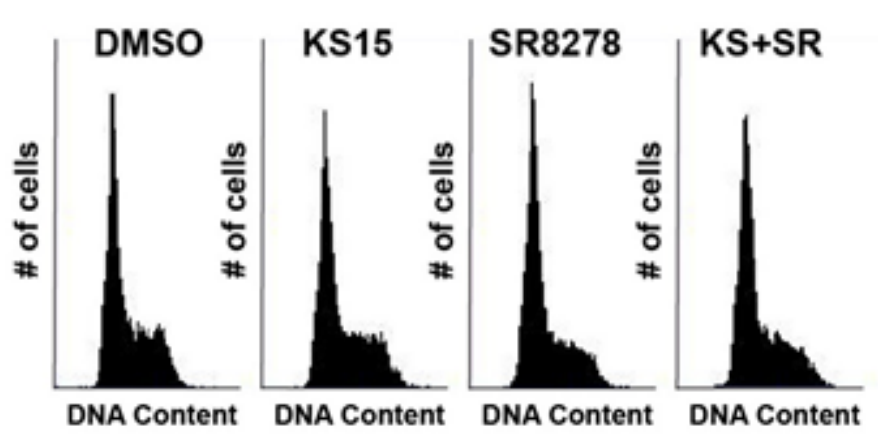

b

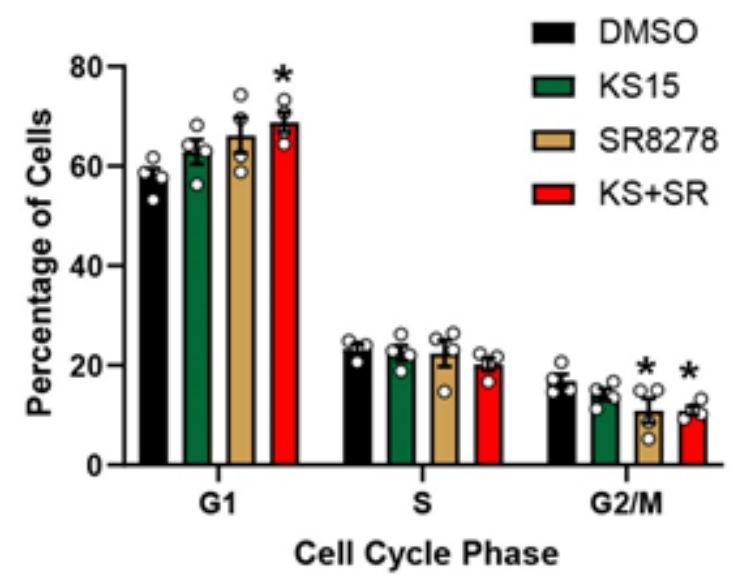

C
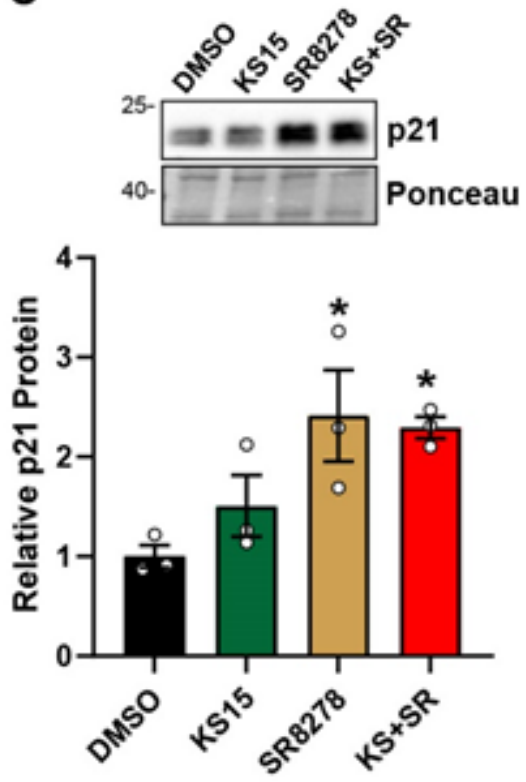

Treatment

\section{Figure 5}

KS15 and SR8278 treatment leads to an increased fraction of cells in G1 phase and to increased

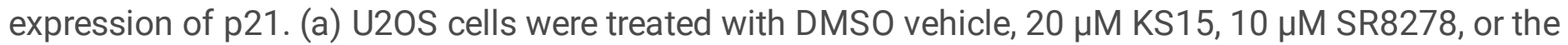
combination of KS15 and SR8278 (KS+SR) for $24 \mathrm{hr}$. Cells were fixed, stained with propidium iodide, and analyzed by flow cytometry. Cell cycle distribution from a representative experiment is shown. (b) Cells were gated for DNA content to determine the fraction of cells in $\mathrm{G} 1, \mathrm{~S}$, and $\mathrm{G} 2 / \mathrm{M}$ phase (average and SEM, n=3). (c) Cell lysates from experiments performed in Figure 3 were analyzed for p21 protein expression (average and SEM, $n=3$ ). The asterisks indicate significant differences in cell cycle distribution and $p 21$ expression ( $p<0.05$; one-way ANOVA). 